\title{
Variability Analysis of Interconnects Terminated by Polynomial Nonlinear Loads
}

\author{
Alessandro Biondi *, Dries Vande Ginste *, Daniël De Zutter *, Paolo Manfredi ${ }^{\dagger}$, Flavio Canavero ${ }^{\dagger}$ \\ * Department of Information Technology, Ghent University, Sint-Pietersnieuwstraat 41, B-9000 Gent, Belgium. \\ † EMC Group, Dipartimento di Elettronica, Politecnico di Torino, Corso Duca degli Abruzzi 24, 10129 Torino, Italy.
}

\begin{abstract}
In this paper, a stochastic modeling method is presented for the analysis of variability effects, induced by the manufacturing process, on interconnect structures terminated by polynomial nonlinear loads. The technique is based on the solution of the pertinent stochastic Telegrapher's equations in time domain by means of the so-called stochastic Galerkin method in conjunction with a finite-difference time-domain technique, allowing the inclusion of, e.g., nonlinear capacitors at the terminals of the lines. The proposed technique is validated and illustrated with an example, demonstrating its accuracy and efficiency.
\end{abstract}

Index Terms-Transmission line, nonlinear, stochastic Galerkin method, polynomial chaos, finite-difference time-domain, variability analysis, uncertainty

\section{INTRODUCTION}

Nowadays, interest has grown in the development of simulation techniques for the analysis of high-speed interconnects, including the effects of possible uncertainties of the circuit parameters [1], [2]. In fact, due to ever more stringent design specifications, expressed in terms of speed, bandwidth, largescale production and miniaturization, the necessity for the development of simulation techniques that are able to model the effect of such uncertainties become crucial. In the past, the authors of the present contribution have especially focussed on the stochastic modeling of interconnect structures that are affected by uncertainties in their geometrical or material properties [3], [4]. Thanks to the application of Polynomial Chaos (PC) expansions, a modeling strategy was devised that largely outperformed traditional Monte Carlo (MC) analysis. Unfortunately, as the technique developed in [3], [4] is in essence a frequency-domain method, only linear loads, connected to the terminals of the interconnects, could be taken into account. Therefore, further steps have been taken and a technique was devised that allows including loads with arbitrary I-V characteristics [5]. In this contribution, we focus on the variability analysis of interconnects that are terminated by nonlinear loads, described by polynomial I-V characteristics, and we show that they can be dealt with via an alternative, closed-form and exact formulation. The goal is to more efficiently and accurately solve the governing stochastic Telegrapher's equations for transmission lines terminated, e.g, by nonlinear polynomial capacitors. The proposed technique combines the so-called Stochastic Galerkin Method (SGM) [3] with the well-known finite-difference time-domain (FDTD) method [6].
This contribution is organized as follows. In Section II, the proposed formalism is explained starting from the stochastic Telegrapher's equations. The SGM framework is constructed and special attention is devoted to the description of polynomial nonlinear loads and to their FDTD implementation. In Section III, the formalism is validated and illustrated by applying it to the variability analysis of a microstrip line, terminated by a nonlinear capacitor that is described by a polynomial model. Conclusions are presented in Section IV.

\section{THEORY}

\section{A. Stochastic Telegrapher's Equations}

Consider a single uniform transmission line, taking the $z$ axis as the axis of invariance. An example of such a line is reported in Fig. 1 (Section III). The electrical behavior of this line in the time domain is described by the wellknown Telegrapher's equations [7]. Often, due to manifacturing tolerances, one or more geometrical and/or material parameters cannot be described in a deterministic way. Thus, these parameters need to be treated as random variables (RVs), described with a probability density function (PDF), rendering the Telegrapher's equations nondeterministic. Let us now define the new set of stochastic Telegrapher's equations, and for ease of notation, we consider a lossless and dispersionfree line, affected by a single stochastic parameter:

$$
\frac{\partial}{\partial z}\left[\begin{array}{c}
v(z, t, \beta) \\
i(z, t, \beta)
\end{array}\right]=-\left[\begin{array}{cc}
0 & L(\beta) \\
C(\beta) & 0
\end{array}\right] \cdot \frac{\partial}{\partial t}\left[\begin{array}{c}
v(z, t, \beta) \\
i(z, t, \beta)
\end{array}\right],
$$

where $v$ and $i$ represent the voltage and current along the line, and $L$ and $C$ are the per-unit-of-length (p.u.l.) transmission line parameters, i.e. the p.u.l. inductance and capacitance, respectively. The nondeterministic voltage and current depend on the position $z$ along the line and the time $t$, but also on a stochastic parameter $\beta$ of which only the PDF is known. From here on, we denote this PDF of $\beta$ as $W_{\beta}(\beta)$, which is defined on a support $\Gamma \subseteq \mathbb{R}$. Due to the presence of the RV $\beta$, (1) cannot be solved in a straightforward way.

\section{B. Stochastic Galerkin Method (SGM)}

The solution to the stochastic Telegrapher's equations (1) can be obtained by relying on the well-established Stochastic Galerkin Method (SGM). A detailed description of this method in the frequency domain is found in [3], [4]. Here, however, the 
goal is to apply the SGM to transmission lines that are terminated by polynomial nonlinear terminations, and in particular by a nonlinear capacitor (see Section II-C), necessitating a time-domain analysis.

First, we apply the Polynomial Chaos (PC) expansion to the above system of differential equations (2), by rewriting the voltage, current and the p.u.l. parameters as follows:

$$
\begin{aligned}
& v(z, t, \beta)=\sum_{k=0}^{K} v_{k}(z, t) \phi_{k}(\beta), \quad L(\beta)=\sum_{k=0}^{K} L_{k} \phi_{k}(\beta), \\
& i(z, t, \beta)=\sum_{k=0}^{K} i_{k}(z, t) \phi_{k}(\beta), \quad C(\beta)=\sum_{k=0}^{K} C_{k} \phi_{k}(\beta),
\end{aligned}
$$

where each function $\phi_{k}(\beta), k=0, \ldots, K$, represents a polynomial of degree $k$. To obtain an accurate expansion, leading to an adequate solution of the stochastic Telegrapher's equations, the polynomial bases are chosen according to the Wiener-Askey scheme [8], meaning that they are orthogonal w.r.t. to the following inner product:

$$
<\phi_{k}(\beta), \phi_{l}(\beta)>=\int_{\Gamma} \phi_{k}(\beta) \phi_{l}(\beta) W_{\beta}(\beta) d \beta=c_{k} \delta_{k l} .
$$

In the above equation, the weighting function $W_{\beta}(\beta)$ coincides with the PDF of $\beta$, and $\delta_{k l}$ is the Kronecker delta. The scalar number $c_{k}$ simply depends on the scaling of the polynomials. In the remainder of this contribution, we choose the polynomials to be orthonormal, i.e. $c_{k}=1$, $\forall k=0, \ldots, K$ [9]. Thanks to the orthogonality, and since the p.u.l. parameters $L$ and $C$ are known as functions of $\beta$, the expansion coefficients $L_{k}$ and $C_{k}$, are easily computed. Nonetheless, the voltage and current expansion coefficients, i.e. $v_{k}$ and $i_{k}$ resp., $k=0, \ldots, K$, are as yet unknown.

Second, the series (2) are substituted into (1). Then, the result is subjected to a Galerkin testing procedure, meaning that the equations are weighted with the same set of polynomials using the inner product (3). This leads to the following set of equations:

$$
\frac{\partial}{\partial z}\left[\begin{array}{c}
\tilde{\mathbf{v}}(z, t) \\
\tilde{\mathbf{i}}(z, t)
\end{array}\right]=-\left[\begin{array}{cc}
0 & \tilde{\mathbf{L}} \\
\tilde{\overline{\mathbf{C}}} & 0
\end{array}\right] \cdot \frac{\partial}{\partial t}\left[\begin{array}{c}
\tilde{\mathbf{v}}(z, t) \\
\tilde{\mathbf{i}}(z, t)
\end{array}\right],
$$

where $\tilde{\mathbf{v}}$ and $\tilde{\mathbf{i}}$ are $(K+1)$-vectors containing the voltage and current expansion coefficients $v_{k}$ and $i_{k}$, and where $\tilde{\overline{\mathbf{L}}}$ and $\tilde{\mathbf{C}}$ are $(K+1) \times(K+1)$-matrices, with matrix elements $\tilde{L}_{m l}=\sum_{k=0}^{K} L_{k} \alpha_{k l m}$ and $\tilde{C}_{m l}=\sum_{k=0}^{K} C_{k} \alpha_{k l m}$ where $\alpha_{k l m}=<\phi_{k}(\beta) \phi_{l}(\beta), \phi_{m}(\beta)>(l, m=0, \ldots, K)$. At this point, thanks to the SGM, we have transformed a set of two stochastic equations (1) into an augmented set of $2(K+1)$ deterministic equations (4). Indeed, in (4), the dependency on $\beta$ has vanished at the cost of an increased number of unknowns, being the voltage and currents expansion coefficients $v_{k}$ and $i_{k}$. Additionally, it is worth mentioning that the augmented equations (4) have exactly the same shape as a classical set of Telegrapher's equations for $K+1$ lines. From the above PC-representation, statistical information such as the mean and the standard deviation of a voltage waveform $v(z, t, \beta)(2)$, as well as PDFs and cumulative distribution functions (CDF), are readily computed [5].

\section{Boundary Conditions}

The $(K+1)$ unknown expansion coefficients $v_{k}$ and $i_{k}$, $k=0, \ldots, K$, are found from (4) by using standard mathematical methods. Nevertheless, a proper set of $(K+1)$ boundary conditions (BCs) needs to be constructed first. These BCs evolve from the generators and loads attached to the terminals of the original stochastic line (1). In this contribution, we focus on the inclusion of nonlinear polynomial loads within the SGM framework. An accurate, but numerical, formulation for arbitrary nonlinearities has been proposed in [5]. Consider a deterministic nonlinear load at the far-end $z=\mathcal{L}$ of the line, described by a capacitance that is a polynomial function of degree $N$ of the voltage across it :

$$
i(\mathcal{L}, t, \beta)=C(v(\mathcal{L}, t, \beta)) \frac{\partial}{\partial t} v(\mathcal{L}, t, \beta),
$$

with

$$
C(v(\mathcal{L}, t, \beta))=\sum_{n=0}^{N} C_{n}(v(\mathcal{L}, t, \beta))^{n},
$$

Now, we apply the PC expansions (2) to (5):

$$
\begin{aligned}
\sum_{k=0}^{K} i_{k}(\mathcal{L}, t) \phi_{k}(\beta)= & \sum_{n=0}^{N} C_{n}\left(\sum_{k=0}^{K} v_{k}(\mathcal{L}, t) \phi_{k}(\beta)\right)^{n} \\
& \times \sum_{j=0}^{K} \frac{\partial}{\partial t} v_{j}(\mathcal{L}, t) \phi_{j}(\beta)
\end{aligned}
$$

By using the multinomial coefficient theorem, (7) becomes:

$$
\begin{aligned}
\sum_{k=0}^{K} i_{k}(\mathcal{L}, t) \phi_{k}(\beta) & =\sum_{j=0}^{K} \sum_{n=0}^{N} \sum_{k_{0}+k_{1}+\ldots+k_{K}=n} C_{n} \\
& \times\left(\begin{array}{c}
n \\
k_{0}, k_{1}, \ldots, k_{K}
\end{array}\right) \prod_{0 \leq r \leq K} \phi_{r}(\beta)^{k_{r}} \phi_{j}(\beta) \\
& \times \prod_{0 \leq r \leq K}\left(v_{r}(\mathcal{L}, t)\right)^{k_{r}} \frac{\partial}{\partial t} v_{j}(\mathcal{L}, t)
\end{aligned}
$$

with the multinomial coefficient, where $k_{0}, k_{1}$, etc are integers

$$
\left(\begin{array}{c}
n \\
k_{0}, k_{1}, \ldots, k_{K}
\end{array}\right)=\frac{n !}{k_{0} ! k_{1} ! \ldots k_{K} !}
$$

Galerkin weighting of (8) finally yields the $K+1$ new BCs, $\forall m=0, \ldots, K$ :

$$
i_{m}(\mathcal{L}, t)=\sum_{j=0}^{K} \tilde{C}_{m j}\left(\mathcal{L}, t, v_{0}, \ldots, v_{K}\right) \frac{\partial}{\partial t} v_{j}(\mathcal{L}, t),
$$

where

$$
\begin{aligned}
& \tilde{C}_{m j}\left(\mathcal{L}, t, v_{0}, \ldots, v_{K}\right)=\sum_{n=0}^{N} \sum_{k_{0}+\ldots+k_{K}=n} C_{n}\left(\begin{array}{c}
n \\
k_{0}, k_{1}, \ldots, k_{K}
\end{array}\right) \\
& \times<\prod_{0 \leq r \leq K}\left(\phi_{r}(\beta)\right)^{k_{r}} \phi_{j}(\beta), \phi_{m}(\beta)>\prod_{0 \leq r \leq K}\left(v_{r}(\mathcal{L}, t)\right)^{k_{r}}
\end{aligned}
$$


It is worth noticing that the factor $C_{n}\left(\begin{array}{c}n \\ k_{0}, k_{1}, \ldots, k_{K}\end{array}\right) \times$ $<\prod_{0 \leq r \leq K}\left(\phi_{r}(\beta)\right)^{k_{r}} \phi_{j}(\beta), \phi_{m}(\beta)>$ is merely a real number. Therefore, despite the somewhat bulky equation (11), the new BCs are relatively simple and can be easily implemented in the SGM framework (see further). Also, in contrast to [5], the $\mathrm{BCs}$ are obtained in an analytical way, making them exact. The derivation of the $\mathrm{BCs}$ for, e.g, a polynomial conductance can be obtained in a similar way.

\section{Implementation via the Finite-Difference Time-Domain (FDTD) Method}

The solution to the augmented set of equations (4) in conjunction with the set of nonlinear BCs (11) is obtained by adopting a finite-difference time-domain (FDTD) technique for transmission lines [7]. For a detailed explanation of the FDTD scheme, we refer the reader to [7], [5]. After discretization of the Telegrapher's equations (4), the following typical FDTD-leapfrog scheme is obtained:

$$
\begin{aligned}
& \tilde{\mathbf{v}}_{p}^{q+1}=\tilde{\mathbf{v}}_{p}^{q}-\frac{\Delta t}{\Delta z} \tilde{\overline{\mathbf{C}}}^{-1} \cdot\left(\tilde{\mathbf{i}}_{p+\frac{1}{2}}^{q+\frac{1}{2}}-\tilde{\mathbf{i}}_{p-\frac{1}{2}}^{q+\frac{1}{2}}\right), \\
& \tilde{\mathbf{i}}_{p+\frac{1}{2}}^{q+\frac{3}{2}}=\tilde{\mathbf{i}}_{p+\frac{1}{2}}^{q+\frac{1}{2}}-\frac{\Delta t}{\Delta z} \tilde{\overline{\mathbf{L}}}^{-1} \cdot\left(\tilde{\mathbf{v}}_{p+1}^{q+1}-\tilde{\mathbf{v}}_{p}^{q+1}\right),
\end{aligned}
$$

where the vectors $\tilde{\mathbf{v}}_{p}^{q}$ stem from the discretization of the voltage waveforms $\mathbf{v}_{k}(z, t)$ at discrete nodes $z_{p}=p \Delta z$, $p=0, \ldots, N_{z}$, and at times $q \Delta t, q=0, \ldots, N_{t}$. The current waveforms $\mathbf{i}_{k}(z, t)$ are assessed at nodes $z_{p}=\left(p+\frac{1}{2}\right) \Delta z$, $p=0, \ldots, N_{z}-1$, and at times $\left(q+\frac{1}{2}\right) \Delta t, q=0, \ldots, N_{t}-1$. The voltages and currents are solved by iterating $p$ for a fixed time (recursively solving first (12) and second (13)), and then iterating time. All voltage and current variables are treated in this way, except for the voltages at the terminals $z=0(p=0)$ and $z=\mathcal{L}\left(p=N_{z}\right)$, for which special update functions need to be constructed. It is readily proven that these are given by:

$$
\begin{aligned}
& \tilde{\mathbf{v}}_{0}^{q+1}=\tilde{\mathbf{v}}_{0}^{q}-\frac{2 \Delta t}{\Delta z} \tilde{\mathbf{C}}^{-1} \cdot\left(\tilde{\mathbf{i}}_{0}^{q+\frac{1}{2}}-\tilde{\mathbf{i}}_{\mathrm{ne}}^{q+\frac{1}{2}}\right), \\
& \tilde{\mathbf{v}}_{N_{z}}^{q+1}=\tilde{\mathbf{v}}_{N_{z}}^{q}-\frac{2 \Delta t}{\Delta z} \tilde{\mathbf{C}}^{-1} \cdot\left(\tilde{\mathbf{i}}_{\mathrm{fe}}^{q+\frac{1}{2}}-\tilde{\mathbf{i}}_{N_{z}-1}^{q+\frac{1}{2}}\right),
\end{aligned}
$$

where the vectors $\mathbf{i}_{\text {ne }}^{q+\frac{1}{2}}$ and $\mathbf{i}_{\text {fe }}^{q+\frac{1}{2}}$ contain the $(K+1)$ currents flowing through the terminals at the near-end $z=0$ and the far-end $z=\mathcal{L}$ respectively. For a nonlinear capacitor, attached to the far-end of the line, the augmented $\mathrm{BC}$ in its discretized form, is given by

$$
\tilde{i}_{f e, m}^{q+\frac{1}{2}}=\sum_{j=0}^{K} \tilde{C}_{m j} \frac{\tilde{v}_{N_{z, m}}^{q+1}-\tilde{v}_{N_{z, m}}^{q}}{2 \Delta t}
$$

where the coefficients $\tilde{C}_{m j}$ are given by (11). Next, the augmented BC are handled within our FDTD scheme implemented in Matlab, in particular making use of the fsolve.m routine, which allows to find the roots of a nonlinear equation. A fast convergence of the iterative solution is assured when the fsolve. $m$ routine is adopted. Indeed, to find the update $\tilde{\mathbf{v}}_{N_{z}}^{q+1}$, we seed the solver with the previous voltage $\tilde{\mathbf{v}}_{N_{z}}^{q}$, which turns out to be an excellent initial value.

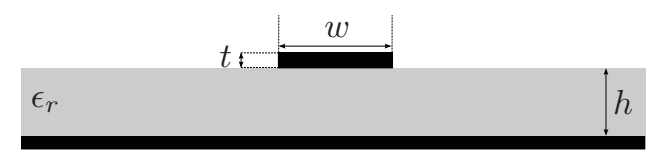

(a) Cross-section of the source-line-load configuration (Fig. 1(b)), where $w=100 \mu \mathrm{m}, h=500 \mu \mathrm{m}$ and $t=35 \mu \mathrm{m}$. The width $w$ of the line and the relative permittivity $\epsilon_{r}$ of the substrate are stochastic parameters.

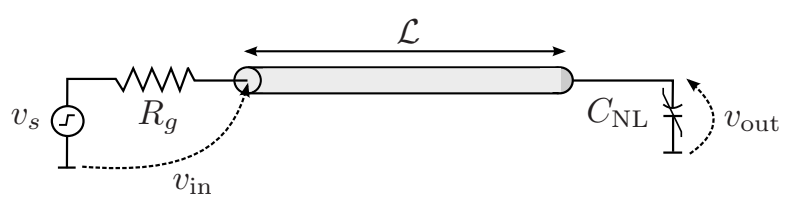

(b) Source-line-load configuration, where $\mathcal{L}=5 \mathrm{~cm}, v_{s}(t)$ is a finite voltage step, $R_{g}=50 \Omega$, and with a nonlinear capacitor $C_{\mathrm{NL}}$.

Fig. 1: Microstrip line under study.

\section{NumERICAL RESUlts}

In this section, the proposed approach is validated and illustrated by applying it to the variability analysis of a single PEC microstrip line. The results shown below have been obtained performing a set of simulations using an Intel(R) i7 (TM) QuadCore 2600K, with a clock speed of $3.4 \mathrm{GHz}$ and 16 GB of RAM. The cross-section of the analyzed structure is presented in Fig. 1(a), while the source-line-load configuration is reported in Fig. 1(b), where $w=100 \mu \mathrm{m}, h=500 \mu \mathrm{m}$ and $t=35 \mu \mathrm{m}$. The relative permittivity $\epsilon_{r}$ of the substrate and the line width $w$ are considered to be two Gaussian RVs with mean $\mu_{\epsilon_{r}}=4$ and $\mu_{w}=100 \mu \mathrm{m}$, respectively, and with normalized standard deviations $\sigma_{\epsilon_{r}}=5 \%$ and $\sigma_{w}=5 \%$. The microstrip line is excited by means of a voltage source $v_{s}(t)$ that produces a finite step, going from $0 \mathrm{~V}$ to $1 \mathrm{~V}$ in a risetime of $50 \mathrm{ps}$. The generator impedance is $R_{g 1}=50 \Omega$. The nonlinear capacitance $C_{\mathrm{NL}}$ is characterized by (6) with $N=2$ and with the following polynomial coefficients: $C_{0}=1 p F, C_{1}=-3 p F / V, C_{2}=5 p F / V^{2}$. Such a nonlinear capacitor could, e.g., represent a MOSFET's gate capacitance, but of course, any polynomial model can be handled with the proposed technique.

In order to obtain a reference result, a set of 10000 Monte Carlo (MC) simulations was performed. The obtained results are also compared to the ones computed using the purely numerical approach proposed in [5], i.e. without exploiting the polynomial behavior of the nonlinear capacitor. In Fig. 2 the mean of the voltage at the output obtained using the two PCbased methods (analytical and numerical) is compared with the result of the reference MC method. For clarity of the figure, results are only shown up to $2 \mathrm{~ns}$. Next, the comparison between the standard deviation computed by means of the proposed method, the MC reference and the numerical solution [5] is reported in Fig. 3. We observe an excellent agreement between all the results. Moreover, the total runtime for the $\mathrm{MC}$ analysis was $41880 \mathrm{~s}$, whereas the novel SGM simulation only took $45 \mathrm{~s}$. By using the SGM technique presented in [5], the total runtime was $79 \mathrm{~s}$. Hence, the proposed technique provides a 


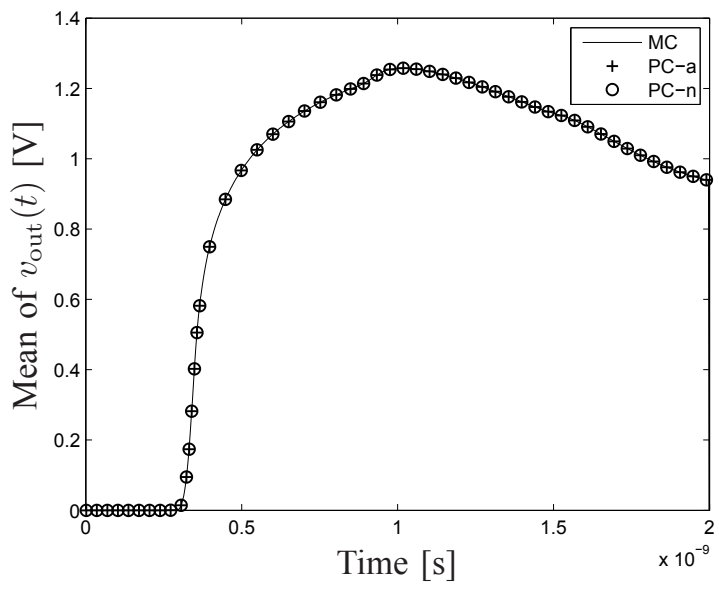

Fig. 2: Mean of the voltage waveform $v_{\text {out }}(t)$, at the terminal of the microstrip line of Fig. 1 obtained with MC and PC. Continuous line: $\mathrm{MC}$ technique; Crosses $(+)$ : newly proposed PC analytical technique (PC-a); Circles (o): numerical PC technique (PC-n) [5]. For clarity, results are only shown up to $2 \mathrm{~ns}$.

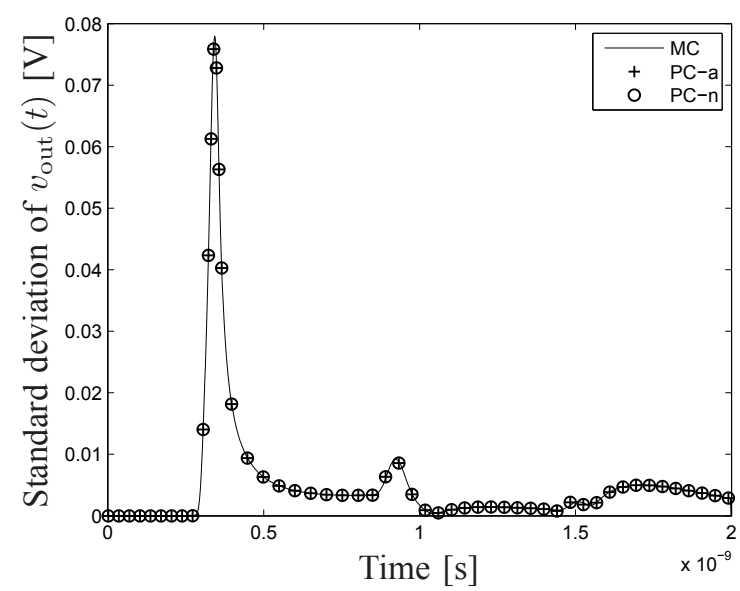

Fig. 3: Standard deviation of $v_{\text {out }}(t)$, obtained with $\mathrm{MC}$ and PC. Continuous line: MC technique; Crosses (+): newly proposed PC analytical technique (PC-a); Circles (o): numerical PC technique (PC-n) [5]. For clarity, results are only shown up to $2 \mathrm{~ns}$.

speedup of ca. $930 \times$ compared to the reference MC method, and, by analytically exploiting the polynomial characteristic of the load, it also outperforms the more general, but purely numerical, technique presented in [5]. Further, we present the cumulative distribution function (CDF) of the maximum of the output voltage, reported in Fig. 4. Apart from the excellent agreement between $\mathrm{MC}$ and the new technique, from this figure, the maximum overshoot that one can expect, is readily assessed.

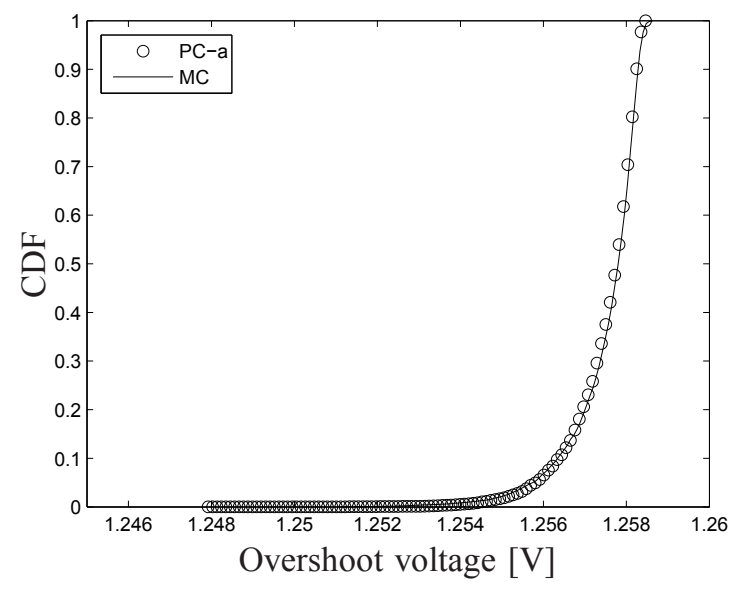

Fig. 4: Cumulative distribution function of $\max \left(v_{\text {out }}(t)\right)$

\section{CONCLUSIONS}

In this contribution we have presented a novel PC-based technique for stochastic circuits dealing with nonlinear terminations described by a polynomial I-V characteristic. The technique is based on combining the SGM with FDTD. It was validated and illustrated by means of an application example, consisting of a microstrip line exhibiting variability of its geometrical and material parameters, and terminated with a nonlinear polynomial capacitance. The results have been compared to a standard and robust Monte Carlo analysis, and to the numerical method presented in [5]. This comparison shows an excellent agreement, and as expected, an improved efficiency in comparison to both $\mathrm{MC}$ and the numerical method.

\section{REFERENCES}

[1] T. Mikazuki and N. Matsui, "Statistical design techniques for high-speed circuit boards with correlated distributions," IEEE Trans. Comp. Pack. \& Man. Tech. Part A, vol. 17, no. 1, pp. 159-165, Mar. 1994.

[2] A. H. Zaabab, Q.-J. Zhang, and M. Nakhla, "A neural network modeling approach to circuit optimization and statistical design," IEEE Trans. Microw. Theory Tech., vol. 43, no. 6, pp. 1349-1358, Jun. 1995.

[3] I. S. Stievano, P. Manfredi, and F. G. Canavero, "Parameters variability effects on multiconductor interconnects via Hermite polynomial chaos," IEEE Trans. on Components, Packaging and Manufacturing Technology, vol. 1, no. 8, pp. 1234-1239, Aug. 2011.

[4] D. Vande Ginste, D. De Zutter, D. Deschrijver, T. Dhaene, P. Manfredi, and F. Canavero, "Stochastic modeling based variability analysis of onchip interconnects," IEEE Transactions on Components, Packaging and Manufacturing Technology, vol. 2, no. 7, pp. 1182-1192, Jul. 2012.

[5] A. Biondi, D. Vande Ginste, D. De Zutter, P. Manfredi, and F. G. Canavero, "Variability analysis of interconnects terminated by general nonlinear loads," IEEE Trans. on Components, Packaging and Manifacturing Technology, vol. 3, no. 7, pp. 1244-1251, Jul. 2013.

[6] A. Taflove, The Finite-Difference Time Domain Method. Norwood: Artech House, 1995.

[7] C. R. Paul, Analysis of Multiconductor Transmission Lines. John Wiley \& Sons, 1994.

[8] D. Xiu and G. E. Karniadakis, "The Wiener-Askey polynomial chaos for stochastic differential equations," SIAM J. Sci. Comput., vol. 24, no. 2, pp. 619-644, 2002

[9] P. Manfredi, D. Vande Ginste, D. De Zutter, and F. G. Canavero, "Improved polynomial chaos discretization schemes to integrate interconnects into design environments," IEEE Microwave and Wireless Components Letters, vol. 23, no. 3, pp. 116-118, Mar. 2013. 